\title{
ASYMPTOTIC EXPANSION OF SOLUTION TO SINGULARLY PERTURBED OPTIMAL CONTROL PROBLEM WITH CONVEX INTEGRAL QUALITY FUNCTIONAL WITH TERMINAL PART DEPENDING ON SLOW AND FAST VARIABLES
}

\section{A.R. DANILIN, A.A. SHABUROV}

Abstract. We consider an optimal control problem with a convex integral qua
tional for a linear system with fast and slow variables in the class of piecewise
controls with smooth constraints on the control
\[ \left\{\begin{array}{l}\dot{x}_{\varepsilon}=A_{11} x_{\varepsilon}+A_{12} y_{\varepsilon}+B_{1} u, \quad t \in[0, T], \quad\|u\| \leqslant 1, \\ \varepsilon \dot{y}_{\varepsilon}=A_{22} y_{\varepsilon}+B_{2} u, \quad x_{\varepsilon}(0)=x^{0}, \quad y_{\varepsilon}(0)=y^{0}, \quad \nabla \varphi_{2}(0)=0, \\ J(u):=\varphi_{1}\left(x_{\varepsilon}(T)\right)+\varphi_{2}\left(y_{\varepsilon}(T)\right)+\int_{0}^{T}\|u(t)\|^{2} d t \rightarrow \text { min, }\end{array}\right. \]

where $x \in \mathbb{R}^{n}, y \in \mathbb{R}^{m}, u \in \mathbb{R}^{r} ; A_{i j}$ and $B_{i}, i, j=1,2$, are constant matrices of corresponding dimension, and the functions $\varphi_{1}(\cdot), \varphi_{2}(\cdot)$ are continuously differentiable in $\mathbb{R}^{n}, \mathbb{R}^{m}$, strictly convex, and cofinite in the sense of the convex analysis. In the general case, for such problem, the Pontryagin maximum principle is a necessary and sufficient optimality condition and there exist unique vectors $l_{\varepsilon}$ and $\rho_{\varepsilon}$ determining an optimal control by the formula

$$
u_{\varepsilon}(T-t):=\frac{C_{1, \varepsilon}^{*}(t) l_{\varepsilon}+C_{2, \varepsilon}^{*}(t) \rho_{\varepsilon}}{S\left(\left\|C_{1, \varepsilon}^{*}(t) l_{\varepsilon}+C_{2, \varepsilon}^{*}(t) \rho_{\varepsilon}\right\|\right)},
$$

where

$$
\begin{aligned}
& C_{1, \varepsilon}^{*}(t):=B_{1}^{*} e^{A_{11}^{*} t}+\varepsilon^{-1} B_{2}^{*} \mathcal{W}_{\varepsilon}^{*}(t), \quad C_{2, \varepsilon}^{*}(t):=\varepsilon^{-1} B_{2}^{*} e^{A_{22}^{*} t / \varepsilon}, \\
& \mathcal{W}_{\varepsilon}(t):=e^{A_{11} t} \int_{0}^{t} e^{-A_{11} \tau} A_{12} e^{A_{22} \tau / \varepsilon} d \tau, \quad S(\xi):= \begin{cases}2, & 0 \leqslant \xi \leqslant 2, \\
\xi, & \xi>2 .\end{cases}
\end{aligned}
$$

The main difference of our problem from the previous papers is that the terminal part of quality functional depends on the slow and fast variables and the controlled system is a more general form. We prove that in the case of a finite number of control change points, a power asymptotic expansion can be constructed for the initial vector of dual state $\lambda_{\varepsilon}=\left(l_{\varepsilon}^{*} \rho_{\varepsilon}^{*}\right)^{*}$, which determines the type of the optimal control.

Keywords: optimal control, singularly perturbed problems, asymptotic expansion, small parameter.

Mathematical Subject Classification: 49N05, 93C70

A.R. Danilin, A.A. Shaburov, Asymptotic expansion of Solution to singularly perturbed OPTIMAL CONTROL PROBLEM WITH CONVEX INTEGRAL QUALITY FUNCTIONAL WITH TERMINAL PART ON SLOW AND FAST VARIABLES.

CDanilin A.R., Shaburov A.A. 2019. 


\section{INTRODUCTION}

The paper is devoted to studying the asymptotics of the vector of the dual state in the problem of optimal control [1, 2, 3] of linear system with fast and slow variables, see survey [4], with an convex integral quality functional [3, Ch. 3] and smooth geometric constraints on a control.

In [5, 6], there were considered problems related with a limiting problem for problems of optimal control by a linear system with fast and slow variables. For other formulation, the asymptotics of solutions of perturbed control problem were considered in [7]-[9]. We note that a controlled system of our form but with a terminal quality functional depending on slow variables only was considered in [8].

In the present work we obtain a complete asymptotic expansion of the vector of dual system determining the optimal control. The main difference of our problem in comparison with that considered in [10] is the dependence of the terminal part of the control functional not only on slow variables but also on fast ones.

\section{Formulation OF PROBLEM AND MAIN RELATIONS} lem:

In the class of piece-wise continuous controls we consider the following optimal control prob-

$$
\left\{\begin{array}{l}
\dot{x}_{\varepsilon}=A_{11} x_{\varepsilon}+A_{12} y_{\varepsilon}+B_{1} u, \quad t \in[0, T], \quad\|u\| \leqslant 1, \\
\varepsilon \dot{y}_{\varepsilon}=A_{22} y_{\varepsilon}+B_{2} u, \quad x_{\varepsilon}(0)=x^{0}, \quad y_{\varepsilon}(0)=y^{0}, \quad \nabla \varphi_{2}(0)=0, \\
J(u):=\varphi_{1}\left(x_{\varepsilon}(T)\right)+\varphi_{2}\left(y_{\varepsilon}(T)\right)+\int_{0}^{T}\|u(t)\|^{2} d t \rightarrow \text { min, }
\end{array}\right.
$$

where $x_{\varepsilon} \in \mathbb{R}^{n}, y_{\varepsilon} \in \mathbb{R}^{m}, u \in \mathbb{R}^{r} ; A_{i j}, B_{i}, i, j=1,2$, are constant matrices of an appropriate dimension and $\varphi_{1}(\cdot), \varphi_{2}(\cdot)$ are continuously differentiable on $\mathbb{R}^{n}$ and $\mathbb{R}^{m}$ functions strictly convex and cofinite in the sense of the convex analysis [11, Sect. 13]. All spaces $\mathbb{R}^{n}, \mathbb{R}^{m}, \mathbb{R}^{r}$ are equipped with the Euclidean norm, which is everywhere denoted by the same symbol $\|\cdot\|$. We note that the terminal part of the quality functional depends on slow and fast variables.

For each fixed $\varepsilon>0$, the controlled system and the quality functional in problem (1) are of the form:

$$
\left\{\begin{array}{l}
\dot{z}_{\varepsilon}=\mathcal{A}_{\varepsilon} z_{\varepsilon}+\mathcal{B}_{\varepsilon} u, \quad t \in[0, T] \\
z_{\varepsilon}(0)=z^{0}, \quad\|u\| \leqslant 1 \\
J(u):=\varphi\left(z_{\varepsilon}(T)\right)+\int_{0}^{T}\|u(t)\|^{2} d t \rightarrow \min
\end{array}\right.
$$

where

$$
\begin{aligned}
& z_{\varepsilon}(t)=\left(\begin{array}{l}
x_{\varepsilon}(t) \\
y_{\varepsilon}(t)
\end{array}\right), \quad z_{\varepsilon}(0):=z^{0}=\left(\begin{array}{c}
x^{0} \\
y^{0}
\end{array}\right), \quad \varphi\left(z_{\varepsilon}(T)\right):=\varphi_{1}\left(x_{\varepsilon}(T)\right)+\varphi_{2}\left(y_{\varepsilon}(T)\right), \\
& \mathcal{A}_{\varepsilon}=\left(\begin{array}{cc}
A_{11} & A_{12} \\
0 & \varepsilon^{-1} A_{22}
\end{array}\right), \quad \mathcal{B}_{\varepsilon}=\left(\begin{array}{c}
B_{1} \\
\varepsilon^{-1} B_{2}
\end{array}\right) .
\end{aligned}
$$

We observe that in the considered convex integral quality functional $J$, the terminal part can be interpreted as a penalty for the error of the control at the final moment of time $T$, while the second part reflect the energy spent for the realization of the control.

We shall say that a pair of matrices $(A, B)$ is completely controllable if the system $\dot{x}=$ $A x+B u$ is controllable. 
Assumption 1. For all sufficiently small $\varepsilon>0$, the pair $\left(\mathcal{A}_{\varepsilon}, \mathcal{B}_{\varepsilon}\right)$ is completely controllable, that is, $\operatorname{rank}\left(\mathcal{B}_{\varepsilon}, \mathcal{A}_{\varepsilon} \mathcal{B}_{\varepsilon}, \ldots, \mathcal{A}_{\varepsilon}^{n+m-1} \mathcal{B}_{\varepsilon}\right)=n+m$.

Assumption 2. All eigenvalues of the matrix $A_{22}$ have negative real parts.

Under Assumption 1, the Pontryagin maximum principle is necessary and sufficient condition of the optimality giving the unique solution of problem (1) [3, Sect. 3.5, Thm. 14].

It was shown in [10, Prop. 1, Eq. (1.6)] that the function $u_{\varepsilon}(t)$ is the only optimal control in problem (1), it is of the form

$$
u_{\varepsilon}(T-t):=\frac{\mathcal{B}_{\varepsilon}{ }^{*} e^{\mathcal{A}_{\varepsilon}^{*} t} \lambda_{\varepsilon}}{S\left(\left\|\mathcal{B}_{\varepsilon}{ }^{*} e^{\mathcal{A}_{\varepsilon}^{*} t} \lambda_{\varepsilon}\right\|\right)}, \quad S(\xi):= \begin{cases}2, & 0 \leqslant \xi \leqslant 2, \\ \xi, & \xi>2,\end{cases}
$$

and the vector $\lambda_{\varepsilon}$ is the unique solution (in view of the cofiniteness of the function $\varphi$; [11, Thm. 26.6]) of the equation

$$
\nabla \varphi^{*}(-\lambda)=e^{\mathcal{A}_{\varepsilon} T} z^{0}+\int_{0}^{T} e^{\mathcal{A}_{\varepsilon} \tau} \mathcal{B}_{\varepsilon} \frac{\mathcal{B}_{\varepsilon}^{*} e^{\mathcal{A}_{\varepsilon}^{*} \tau} \lambda}{S\left(\left\|\mathcal{B}_{\varepsilon}^{*} e^{\mathcal{A}_{\varepsilon}^{*} \tau} \lambda\right\|\right)} d \tau
$$

Here $\nabla \varphi^{*}$ is the gradient of the function $\varphi^{*}$ dual to the function $\varphi$ in the sense of the convex analysis, see [11, Sect. 12].

We note that in the considered case

$$
\varphi^{*}(\lambda)=\varphi_{1}^{*}(l)+\varphi_{2}^{*}(\rho) \quad \text { and } \quad \nabla \varphi_{2}^{*}(0)=0 .
$$

We shall consider the vector $\lambda_{\varepsilon}$ determining the optimal control in problem 11 as $\lambda_{\varepsilon}=\left(\begin{array}{c}l_{\varepsilon} \\ \rho_{\varepsilon}\end{array}\right)$, where $l_{\varepsilon} \in \mathbb{R}^{n}, \rho_{\varepsilon} \in \mathbb{R}^{m}$.

Straightforward calculation of the matrix exponent of the controlled system in problem (1) gives:

$$
e^{\mathcal{A}_{\varepsilon} t}:=\left(\begin{array}{cc}
e^{A_{11} t} & \mathcal{W}_{\varepsilon}(t) \\
0 & e^{\frac{A_{22} t}{\varepsilon}}
\end{array}\right)
$$

where $\mathcal{W}_{\varepsilon}^{\prime}(t)=A_{11} \mathcal{W}_{\varepsilon}(t)+A_{12} e^{\frac{A_{22} t}{\varepsilon}}$ and $\mathcal{W}_{\varepsilon}(0)=0$. This is why

$$
\mathcal{W}_{\varepsilon}(t):=e^{A_{11} t} \int_{0}^{t} e^{-A_{11} \tau} A_{12} e^{\frac{A_{22} \tau}{\varepsilon}} d \tau
$$

Integrating by parts in the right hand side in identity (6), we obtain

$$
\mathcal{W}_{\varepsilon}(t)=\varepsilon\left(A_{12} e^{\frac{A_{22} t}{\varepsilon}}-e^{A_{11} t} A_{12}\right) A_{22}^{-1}+\varepsilon A_{11} \mathcal{W}_{\varepsilon}(t) A_{22}^{-1},
$$

and by the boundedness of $A_{12} e^{\frac{A_{22} t}{\varepsilon}}-e^{A_{11} t} A_{12}$ on $[0, T]$,

$$
\mathcal{W}_{\varepsilon}(t)=\varepsilon \sum_{k=0}^{\infty} \varepsilon^{k} A_{11}^{k}\left(A_{12} e^{\frac{A_{22} t}{\varepsilon}}-e^{A_{11} t} A_{12}\right) A_{22}^{-(k+1)}
$$

We shall make use of the following notation:

$$
C_{\varepsilon}(t)=\left(\begin{array}{c}
C_{1, \varepsilon}(t) \\
C_{2, \varepsilon}(t)
\end{array}\right):=e^{\mathcal{A}_{\varepsilon} t} \mathcal{B}_{\varepsilon}=\left(\begin{array}{c}
e^{A_{11} t} B_{1}+\varepsilon^{-1} \mathcal{W}_{\varepsilon}(t) B_{2} \\
\varepsilon^{-1} e^{\frac{A_{22} t}{\varepsilon}} B_{2}
\end{array}\right)
$$


According identity (4) and notation (8), equation (3) is transformed into the system of equations

$$
\left\{\begin{array}{l}
\nabla \varphi_{1}^{*}\left(-l_{\varepsilon}\right)=e^{A_{11} T} x^{0}+\mathcal{W}_{\varepsilon}(T) y^{0}+\int_{0}^{T} C_{1, \varepsilon}(t) u_{\varepsilon}(T-t) d t, \\
\nabla \varphi_{2}^{*}\left(-\rho_{\varepsilon}\right)=e^{A_{22} T / \varepsilon} y^{0}+\int_{0}^{T} C_{2, \varepsilon}(t) u_{\varepsilon}(T-t) d t,
\end{array}\right.
$$

where

$$
u_{\varepsilon}(T-t):=\frac{C_{1, \varepsilon}^{*}(t) l_{\varepsilon}+C_{2, \varepsilon}^{*}(t) \rho_{\varepsilon}}{S\left(\left\|C_{1, \varepsilon}^{*}(t) l_{\varepsilon}+C_{2, \varepsilon}^{*}(t) \rho_{\varepsilon}\right\|\right)}
$$

Definition 1. A limiting problem for problem (1) is

$$
\left\{\begin{array}{l}
\dot{x}_{0}=A_{0} x_{0}+B_{0} u, \quad t \in[0, T], \quad\|u\| \leqslant 1, \\
A_{0}:=A_{11}, \quad B_{0}:=B_{1}-A_{12} A_{22}^{-1} B_{2}, \quad x_{0}(0)=x^{0}, \\
J_{0}(u):=\varphi_{1}\left(x_{0}(T)\right)+\int_{0}^{T}\|u(t)\|^{2} d t \rightarrow \min .
\end{array}\right.
$$

Assumption 3. The pairs of matrices $\left(A_{0}, B_{0}\right),\left(A_{22}, B_{2}\right)$ are completely controllable.

By [5], Assumptions 2 and 3 ensure Assumption 1 for all sufficiently small $\varepsilon$.

Formulae (5), (7) and (8) imply

$$
\begin{aligned}
& C_{1, \varepsilon}(t)=C_{1,0}(t)+A_{12} A_{22}^{-1} e^{\frac{A_{22} t}{\varepsilon}} B_{2}+O(\varepsilon), \quad \varepsilon \rightarrow 0, \quad C_{1,0}(t):=e^{A_{0} t} B_{0}, \\
& \frac{\partial}{\partial t} C_{1, \varepsilon}(t)=\frac{d}{d t} C_{1,0}(t)+\varepsilon^{-1} A_{12} e^{\frac{A_{22} t}{\varepsilon}} B_{2}+A_{11} A_{12} e^{\frac{A_{22} t}{\varepsilon}} A_{22}^{-1} B_{2}+O(\varepsilon), \quad \varepsilon \rightarrow 0,
\end{aligned}
$$

uniformly on the segment $[0, T]$.

We mention the known fact that under Assumption 2 there exist $\gamma>0$ and $K>0$ such that

$$
\left\|e^{\frac{A_{22} t}{\varepsilon}}\right\| \leqslant K e^{-\frac{\gamma t}{\varepsilon}} \text {. }
$$

If a vector function $f_{\varepsilon}(t)$ is such that $f_{\varepsilon}(t)=O\left(\varepsilon^{\alpha}\right)$ as $\varepsilon \rightarrow 0$ for each $\alpha>0$ uniformly in $t \in[a, b]$, we shall write $\mathbb{O}$ instead of $f_{\varepsilon}(t)$. In particular,

$$
\left\|e^{A_{22} t / \varepsilon}\right\|=\mathbb{O}, \quad e^{-\gamma t / \varepsilon}=\mathbb{O} \quad \text { as } t \in\left[\varepsilon^{p}, T\right], \quad p \in(0,1),
$$

where $\gamma>0$.

It follows from formulae (11), (12) and estimate (13) that there exist $K_{1}>0$ and $\varepsilon_{0}>0$ such that for $\varepsilon \in\left(0, \varepsilon_{0}\right)$ and $t \in[\sqrt{\varepsilon}, T]$, the inequalities hold

$$
\left\|C_{1, \varepsilon}^{*}(t)-C_{1,0}^{*}(t)\right\| \leqslant K_{1} \varepsilon, \quad\left\|\frac{\partial}{\partial t} C_{1, \varepsilon}^{*}(t)-\frac{d}{d t} C_{1,0}^{*}(t)\right\| \leqslant K_{1} \varepsilon .
$$

\section{Auxiliary STATEMENTS ON COFinite FUnCTIONS}

According [11, Thm. 26.6], if $f$ is a differentiable strictly convex cofinite function on $\mathbb{R}^{n}$, then $\nabla f: \mathbb{R}^{n} \rightarrow \mathbb{R}^{n}$ is a one-to-one correspondence on $\mathbb{R}^{n}$ and $f^{*}$ is a differentiable strictly convex cofinite function on $\mathbb{R}^{n}$.

Lemma 1. Let $f$ be a differentiable strictly convex cofinite function on $\mathbb{R}^{n}, \mathbb{L}$ be a nonnegative linear operator in $\mathbb{R}^{n}$, that is,

$$
\langle\mathbb{L} l, l\rangle \geqslant 0 \quad \text { for all } \quad l \in \mathbb{R}^{n} .
$$


Then the $g(l)=f(l)+\frac{1}{2}\langle\mathbb{L} l, l\rangle$ is a differentiable strictly convex cofinite function on $\mathbb{R}^{n}$ and $\nabla g(l)=\nabla f(l)+\mathbb{L} l$.

Proof. We begin with proving that $g(l)$ is a differentiable strictly convex cofinite function on $\mathbb{R}^{n}$. We calculate the derivative of the scalar product $\frac{1}{2}\langle\mathbb{L} l, l\rangle$ along the direction of $\Delta l$ :

$$
D\left(\frac{1}{2}\langle\mathbb{L} l, l\rangle\right)(\Delta l)=\left.\frac{\partial}{\partial l}\right|_{t=0} \frac{\langle\mathbb{L}(l+t \Delta l), l+t \Delta l\rangle}{2}=\langle\mathbb{L} l, \Delta l\rangle,
$$

and we obtain that $\nabla\left(\frac{1}{2}\langle\mathbb{L} l, l\rangle\right)=\mathbb{L} l$. According the definition [11], a convex function $f$ is cofinite if the following relation holds:

$$
\lim _{\lambda \rightarrow+\infty} \frac{f(\lambda l)}{\lambda}=+\infty \quad \text { for all } \quad l \neq 0
$$

Let us show that the function $g(l)$ obeys this condition.

For each $\lambda>0$ we have:

$$
\frac{g(\lambda l)}{\lambda}=\frac{f(\lambda l)}{\lambda}+\frac{1}{2} \cdot \frac{\langle\mathbb{L}(\lambda l), \lambda l\rangle}{\lambda}=\frac{f(\lambda l)}{\lambda}+\frac{\lambda}{2} \cdot\langle\mathbb{L} l, l\rangle \geqslant \frac{f(\lambda l)}{\lambda} \rightarrow+\infty \quad \text { as } \quad \lambda \rightarrow+\infty .
$$

Corollary 1. Let a function $f$ satisfies the assumptions of Lemma 1, and $f^{*}$ is a dual function for $f$ in the sense of the convex analysis. Then the equation $\nabla f^{*}(l)+\mathbb{L} l=d$ has the unique solution for each vector $d$.

This corollary follows Lemma 1 and Theorem 26.6 in [11].

\section{Limiting VAlues of Vectors $l_{\varepsilon}$ AND $\rho_{\varepsilon}$}

Theorem 1. Let Assumptions 1 and 2 hold and the vector $\lambda_{\varepsilon}^{*}=\left(\begin{array}{ll}l_{\varepsilon}^{*} & \rho_{\varepsilon}^{*}\end{array}\right)$ is the unique solution of system (9). Then the vectors $l_{\varepsilon}, \rho_{\varepsilon}$ are bounded and

$$
l_{\varepsilon} \rightarrow l_{0} \quad \text { as } \quad \varepsilon \rightarrow+0
$$

where $l_{0}$ is the unique solution of the equation

$$
0=-\nabla \varphi_{1}^{*}(-l)+e^{A_{11} T} x^{0}+\int_{0}^{T} C_{1,0}(t) \frac{C_{1,0}^{*}(t) l}{S\left(\left\|C_{1,0}^{*}(t) l\right\|\right)} d t .
$$

Proof. It is known that at the final time $T$, the set of attainability of the controlled system in problem (1) is bounded uniformly in $\varepsilon \in\left(0, \varepsilon_{0}\right]$, see, for instance, [6, Thm. 3.1]. Hence, the left hand side of equation (3) is bounded. This is why, as $\varepsilon \rightarrow 0$, the quantity $\nabla \varphi^{*}\left(-\lambda_{\varepsilon}\right)$ is bounded as well. Since the function $\varphi^{*}$ is cofinite, according [11, Lm. 26.7], the vector $\lambda_{\varepsilon}$ is bounded. Therefore, the vectors $l_{\varepsilon}, \rho_{\varepsilon}$ are bounded.

We partition the interval of integration in the first identity $(9)$ into two pieces: $[0, \sqrt{\varepsilon}]$ and $[\sqrt{\varepsilon}, T]$. Taking into consideration identity (6) and the notation (8) being representations of matrices $\mathcal{W}_{\varepsilon}(t)$ and $C_{\varepsilon}(t)$ in system $(9)-(10)$, we can write the first identity (9) as

$$
\nabla \varphi_{1}^{*}\left(-l_{\varepsilon}\right)=e^{A_{11} T} x^{0}+O(\sqrt{\varepsilon})+\int_{\sqrt{\varepsilon}}^{T} C_{1, \varepsilon}(t) \frac{C_{1, \varepsilon}^{*}(t) l_{\varepsilon}}{S\left\|C_{1, \varepsilon}^{*}(t) l_{\varepsilon}\right\|} d t \quad \text { as } \quad \varepsilon \rightarrow 0 .
$$


Let $l_{0}$ be an arbitrary limiting point of the function $l_{\varepsilon}$ as $\varepsilon \rightarrow 0$. Passing to the limit as $\varepsilon \rightarrow 0$ in identity $(19)$, by inequalities $(15)$ we obtain the identity

$$
\nabla \varphi_{1}^{*}\left(-l_{0}\right)=e^{A_{11} T} x^{0}+\int_{0}^{T} C_{1,0}(t) \frac{C_{1,0}^{*}(t) l_{0}}{S\left\|C_{1,0}^{*}(t) l_{0}\right\|} d t
$$

that is, $l_{0}$ satisfies equation (18). This equation reads as

$$
\nabla \varphi_{1}^{*}\left(-l_{0}\right)+\mathbb{L}\left(-l_{0}\right)=e^{A_{11} T} x^{0}
$$

and $\mathbb{L} \geqslant 0$. This is why by Corollary 1 of Lemma 1 , this equation possesses the unique solution. Thus, $l_{0}$ is the unique limiting point for $l_{\varepsilon}$ and $l_{\varepsilon} \rightarrow l_{0}$ as $\varepsilon \rightarrow 0$.

Theorem 2. Let the assumptions of Theorem 1 hold, and $B_{2}$ is a mapping of $\mathbb{R}^{r}$ onto $\mathbb{R}^{m}$; in particular, $r \geqslant m$. Then $\rho_{\varepsilon} \rightarrow 0$, the quantity $\left\{r_{\varepsilon}\right\}\left(r_{\varepsilon}:=\varepsilon^{-1} \rho_{\varepsilon}\right)$ is bounded as $\varepsilon \rightarrow+0$ and all its limiting points $r_{0}$ satisfy the equation

$$
0=\int_{0}^{+\infty} e^{A_{22} \tau} B_{2} \frac{B_{0}^{*} l_{0}+B_{2}^{*} e^{A_{22}^{*} \tau}\left(r_{0}+\left(A_{22}^{*}\right)^{-1} A_{12}^{*} l_{0}\right)}{S\left(\left\|B_{0}^{*} l_{0}+B_{2}^{*} e^{A_{22}^{*} \tau}\left(r_{0}+\left(A_{22}^{*}\right)^{-1} A_{12}^{*} l_{0}\right)\right\|\right)} d \tau .
$$

Proof. We change the variable $\tau:=t / \varepsilon$ in the integral in the second identity in system (9). We choose arbitrary $\delta>0$ and taking into consideration estimate (13), we rewrite this identity as

$$
\nabla \varphi_{2}^{*}\left(-\rho_{\varepsilon}\right)=\mathbb{O}+\int_{0}^{\delta} e^{A_{22} \tau} B_{2} \frac{\tilde{B}(\tau, \varepsilon) l_{\varepsilon}+B_{2}^{*} e^{A_{22}^{*} \tau} r_{\varepsilon}}{S\left(\left\|\tilde{B}(\tau, \varepsilon) l_{\varepsilon}+B_{2}^{*} e^{A_{22}^{*} \tau} r_{\varepsilon}\right\|\right)} d \tau+O\left(e^{-\gamma \delta}\right),
$$

where $r_{\varepsilon}:=\rho_{\varepsilon} / \varepsilon$, and

$$
\tilde{B}(\tau, \varepsilon):=B_{0}^{*} e^{A_{11}^{*} \varepsilon \tau}+B_{2}^{*} e^{A_{22}^{*} \tau}\left(A_{22}^{*}\right)^{-1} A_{12}^{*} .
$$

We note that $\tilde{B}(\tau, \varepsilon) l_{\varepsilon} \rightarrow \tilde{B}(\tau, 0) l_{0}$ as $\varepsilon \rightarrow 0$ uniformly on $[0, \delta]$ and $\tilde{B}(\tau, 0)$ is bounded on $[0,+\infty)$.

Let $\rho_{0}$ be an arbitrary limiting point of $\rho_{\varepsilon}$ as $\varepsilon \rightarrow 0$, that is, there exists $\left\{\varepsilon_{k}\right\}$ such that $\varepsilon_{k} \rightarrow 0$ and $\rho_{k}:=\rho_{\varepsilon_{k}} \rightarrow \rho_{0}$.

We assume that $r_{k}:=r_{\varepsilon_{k}}$ is unbounded. Without loss of generality we suppose that

$$
r_{k} \rightarrow \infty, \quad \frac{r_{k}}{\left\|r_{k}\right\|} \rightarrow \bar{r}, \quad\|\bar{r}\|=1, \quad \rho_{0}=\left\|\rho_{0}\right\| \bar{r} .
$$

Since the function $B_{2}^{*} e^{A_{22}^{*} \tau} r$ is jointly continuous in the variable $\tau$ and vector $r$, and as $r \neq 0$, by the injectivity of $B_{2}^{*}$, we have $B_{2}^{*} e^{A_{22}^{*} \tau} r \neq 0$, there exists $K_{0}(\delta)>0$ such that

$$
\left\|B_{2}^{*} e^{A_{22}^{*} \tau} r\right\| \geqslant K_{0}(\delta)\|r\|
$$

for all $r$ and all $\tau \in[0, \delta]$. This is why, by relations $(23)$, for all sufficiently large $k$, the inequality holds:

$$
\left\|C_{1, \varepsilon_{k}}^{*}(\varepsilon \tau) l_{\varepsilon_{k}}+B_{2}^{*} e^{A_{22}^{*} \tau} r_{k}\right\|>2
$$

and identity 21 becomes

$$
\nabla \varphi_{2}^{*}\left(-\rho_{k}\right)=\int_{0}^{\delta} e^{A_{22} \tau} B_{2} \frac{\frac{1}{\left\|r_{k}\right\|} \tilde{B}\left(\tau, \varepsilon_{k}\right) l_{k}+B_{2}^{*} e^{A_{22}^{*} \tau} \frac{r_{k}}{\left\|r_{k}\right\|}}{\left\|\frac{1}{\left\|r_{k}\right\|} \tilde{B}\left(\tau, \varepsilon_{k}\right) l_{k}+B_{2}^{*} e^{A_{22}^{*} \tau} \frac{r_{k}}{\left\|r_{k}\right\|}\right\|} d \tau+\mathbb{O}+O\left(e^{-\gamma \delta}\right) .
$$


We pass to the limit in $k$ and then as $\delta \rightarrow+\infty$ in identity (24). Then in view of relations (23) we obtain the identity:

$$
\nabla \varphi_{2}^{*}\left(-\left\|\rho_{0}\right\| \bar{r}\right)=\int_{0}^{+\infty} e^{A_{22} \tau} B_{2} \frac{B_{2}^{*} e^{A_{22}^{*} \tau} \bar{r}}{\left\|B_{2}^{*} e^{A_{22}^{*} \tau}\right\|} d \tau
$$

We calculate the scalar product of the latter equation with $\bar{r}$ and we obtain:

$$
\left\langle\nabla \varphi_{2}^{*}\left(-\left\|\rho_{0}\right\| \bar{r}\right), \bar{r}\right\rangle=\int_{0}^{+\infty}\left\|B_{2}^{*} e^{A_{22}^{*} \tau} \bar{r}\right\| d \tau
$$

By Assumption 3, the right hand side of the above identity is positive, while the left hand side is non-positive due to the monotonicity of $\nabla \varphi_{2}^{*}$ and the identity $\nabla \varphi_{2}^{*}(0)=0$; this is a contradiction. Thus, $\rho_{\varepsilon} \rightarrow 0$. If $r_{\varepsilon}$ is unbounded, reproducing the above arguing, we arrive at a contradicting inequality similar to (25):

$$
0=\int_{0}^{+\infty}\left\|B_{2}^{*} e^{A_{22}^{*} \tau} \bar{r}\right\| d \tau
$$

Finally, if $r_{0}$ is a limiting point of $r_{\varepsilon}$, then we pass to the limit as $\varepsilon \rightarrow 0$ in 21) and then we pass to the limit as $\delta \rightarrow+\infty$. In view of notation 22 we obtain identity (20).

Theorem 3. Let the assumptions of Theorem 2 holds. Then equation (20) has the unique solution $r_{0}$ and $r_{\varepsilon} \rightarrow r_{0}$.

Proof. We introduce the notations: $l:=B_{0}^{*} l_{0}, r:=r_{0}+\left(A_{22}^{*}\right)^{-1} A_{12}^{*} l_{0}$. Then equation 20 casts into the form:

$$
F(r):=\int_{0}^{+\infty} e^{A_{22} \tau} B_{2} \frac{l+B_{2}^{*} e^{A_{22}^{*} \tau}}{S\left(\| l+B_{2}^{*} e^{A_{22}^{*} \tau} r\right)} d \tau=0 .
$$

If $l=0$, we multiply identity (26) by $r$ and we obtain:

$$
\int_{0}^{+\infty} \frac{\left\|B_{2}^{*} e^{A_{22}^{*} r}\right\|^{2}}{S\left(\| B_{2}^{*} e^{A_{22}^{*} \tau} r\right)} d \tau=0
$$

Since the integrand is continuous and non-negative, we have $\left\|B_{2}^{*} e^{A_{22}^{*} \tau} r\right\| \equiv 0$ and by Assumption 3 this implies $r=0$.

Let $l \neq 0$. Assume that there exist two different solutions $r_{1} \neq r_{2}$ to equation (26): $F\left(r_{1}\right)=$ $F\left(r_{2}\right)=0$. By the Lagrange formula,

$$
0=\left\langle F\left(r_{1}\right)-F\left(r_{2}\right), r_{1}-r_{2}\right\rangle=\left\langle\left.\frac{\partial}{\partial r} F(r)\right|_{r=r^{\prime}}\left(r_{1}-r_{2}\right), r_{1}-r_{2}\right\rangle,
$$

where $r^{\prime} \in\left[r_{1}, r_{2}\right]$. Let us show that as $r_{1} \neq r_{2}$, identity (27) is impossible.

We rewrite the integral in (26) as a sum of two integrals over two sets:

$$
E_{1}(r):=\left\{\tau \in[0,+\infty): \| l+B_{2}^{*} e^{A_{22}^{*} \tau} r \leqslant 2\right\}, \quad E_{2}(r):=\left\{\tau \in[0,+\infty):\left\|l+B_{2}^{*} e^{A_{22}^{*} \tau} r\right\| \geqslant 2\right\} .
$$

Then the integral in the right hand side in equation $(26)$ is split into two integrals:

$$
F(r)=\int_{E_{1}(r)} e^{A_{22} \tau} B_{2} \frac{l+B_{2}^{*} e^{A_{22}^{*} \tau}}{2} d \tau+\int_{E_{2}(r)} e^{A_{22} \tau} B_{2} \frac{l+B_{2}^{*} e^{A_{22}^{*} \tau}}{\| l+B_{2}^{*} e^{A_{22}^{*} \tau} r} d \tau .
$$

Since $B_{2}^{*} e^{A_{22}^{*} \tau} r \rightarrow 0$ as $\tau \rightarrow+\infty$, the sets $E_{1}(r)$ and $E_{2}(r)$ consist of finitely many segments. 
Let us find the derivative $D F\left(r^{\prime}\right)(\Delta r)$ of the function $F$ at the point $r^{\prime}$ along the direction $\Delta r$. We employ representation (28) and the known formula:

$$
D\left(\int_{\alpha(r)}^{\beta(r)} f(t, r) d t\right)(\Delta r)=\int_{\alpha(r)}^{\beta(r)} \frac{\partial f(t, r)}{\partial r}(\Delta r) d t++f(\beta(r), r) \frac{\partial \beta}{\partial r}(\Delta r)-f(\alpha(r), r) \frac{\partial \alpha}{\partial r}(\Delta r) .
$$

Since the integrands coincide at the common points of $E_{1}(r)$ and $E_{2}(r)$, the final formula for $D F$ involves no non-integral terms.

Since

$$
\frac{\partial}{\partial r}\left(e^{A_{22} \tau} B_{2} \frac{l+B_{2}^{*} e^{A_{22}^{*} \tau}}{2}\right)(\Delta r)=C(\tau) \frac{C^{*}(\tau) \Delta r}{2}, \quad C(\tau):=e^{A_{22} \tau} B_{2}
$$

and

$$
\begin{aligned}
\frac{\partial}{\partial r}\left(e^{A_{22} \tau} B_{2}\right. & \frac{l+B_{2}^{*} e^{A_{22}^{*} \tau} r}{\left.\| l+B_{2}^{*} e^{A_{22}^{*} \tau \|}\right)} \\
& =C(\tau) \frac{C^{*}(\tau) \Delta r\left\|l+C^{*}(\tau) r\right\|^{2}-\left\langle C^{*}(\tau) \Delta r, l+C^{*}(\tau) r\right\rangle\left(l+C^{*}(\tau) r\right)}{\left\|l+C^{*}(\tau) r\right\|^{3}}
\end{aligned}
$$

then

$$
\begin{aligned}
& D F\left(r^{\prime}\right)(\Delta r)=D F_{1}\left(r^{\prime}\right)(\Delta r)+D F_{2}\left(r^{\prime}\right)(\Delta r) \\
& D F_{1}\left(r^{\prime}\right)(\Delta r)=\frac{1}{2} \int_{E_{1}\left(r^{\prime}\right)} e^{A_{22} \tau} B_{2} B_{2}^{*} e^{A_{22}^{*} \tau} \Delta r d \tau \\
& D F_{2}\left(r^{\prime}\right)(\Delta r)=\int_{E_{2}\left(r^{\prime}\right)} C(\tau) \frac{C^{*}(\tau) \Delta r\left\|l+C^{*}(\tau) r\right\|^{2}-\left\langle C^{*}(\tau) \Delta r, l+C^{*}(\tau) r\right\rangle\left(l+C^{*}(\tau) r\right)}{\left\|l+C^{*}(\tau) r\right\|^{3}} d \tau .
\end{aligned}
$$

If $E_{1}\left(r^{\prime}\right) \neq \emptyset$, the latter identity in 290 implies $D F_{1}\left(r^{\prime}\right)>0$. It follows from the CauchySchwarz inequality and relations 290 that $D F_{2}\left(r^{\prime}\right) \geqslant 0$. This is why, if $E_{1}\left(r^{\prime}\right) \neq \emptyset$, then $D F\left(r^{\prime}\right)>0$ and identity (27) is possible only as $\Delta r=r_{1}-r_{2}=0$.

Since $\Delta r \neq 0$, it follows from identity (27) that

$$
E_{1}\left(r^{\prime}\right)=\emptyset
$$

and by the Cauchy-Schwarz inequality, the vector $l+B_{2}^{*} e^{A_{22}^{*} \tau} r^{\prime}$ is parallel to the vector $B_{2}^{*} e^{A_{22}^{*} \tau} \Delta r$ for all $\tau$. The identity $E_{1}\left(r^{\prime}\right)=\emptyset$ means that

$$
\left\|l_{1}+e^{A_{22}^{*} \tau} r^{\prime}\right\| \geqslant 2 \text { for all } \tau \text {. }
$$

By the assumptions of the theorem, $B_{2}^{*} e^{A_{22}^{*} \tau} \Delta r \neq 0$. Hence, there exists a function $\beta: \mathbb{R} \rightarrow \mathbb{R}$ such that

$$
l+B_{2}^{*} e^{A_{22}^{*} \tau} r^{\prime}=\beta(\tau) B_{2}^{*} e^{A_{22}^{*} \tau} \Delta r \text { for all } \tau .
$$

Hence, $l$ reads as $B_{2}^{*} l_{1}$. Thus, if $l \notin \operatorname{Im}\left(B_{2}^{*}\right)$, identity (27) is impossible.

By the injectivity of the operator $B_{2}^{*}$ we obtain that

$$
\forall \tau \quad l_{1}+e^{A_{22}^{*} \tau} r^{\prime}=\beta(\tau) e^{A_{22}^{*} \tau} \Delta r .
$$

We multiply identity 31 by $e^{-A_{22}^{*} \tau}$ and we get:

$$
e^{-A_{22}^{*} \tau} l_{1}+r^{\prime}=\beta(\tau) \Delta r
$$

Hence, the function $\beta(\tau)$ is infinitely differentiable. We differentiate identity 32 twice in $\tau$ and we obtain:

$$
-A_{22}^{*} e^{-A_{22}^{*} \tau} l_{1}=\beta^{\prime}(\tau) \Delta r, \quad\left(A_{22}^{*}\right)^{2} e^{-A_{22}^{*} \tau} l_{1}=\beta^{\prime \prime}(\tau) \Delta r .
$$


As $\tau=0$, this gives the identities:

$$
-A_{22}^{*} l_{1}=\beta^{\prime}(0) \Delta r, \quad\left(A_{22}^{*}\right)^{2} l_{1}=\beta^{\prime \prime}(0) \Delta r .
$$

If $\beta^{\prime}(0)=0$ or $\beta^{\prime \prime}(0)=0$, then $l_{1}=0$ that contradicts the assumptions of the theorem.

It follows from identity $(33)$ that

$$
\beta^{\prime \prime}(0) \Delta r=\left(A_{22}^{*}\right)^{2} l_{1}=-A_{22}^{*} \beta^{\prime}(0) \Delta r,
$$

that is, the vector $\Delta r$ is an eigenvector of the matrix $A_{22}^{*}$. Hence,

$$
A_{22}^{*} \Delta r=-\alpha \Delta r, \quad \alpha>0,
$$

where $\alpha=\beta^{\prime \prime}(0) / \beta^{\prime}(0)$ is an eigenvalue of the matrix $A_{22}^{*}$. If the matrix $A_{22}^{*}$ has no real eigenvalues, identity (27) is impossible.

It follows from identities (33) and (34) that the vector $l_{1}$ is parallel to the vector $\Delta r$. This is why by identity (32) and $r^{\prime}$ is parallel to the vector $l_{1}$. Since $r^{\prime}=r_{1}-\beta_{0} \Delta r$ for some $\beta_{0}$, it follows that the vectors $r_{1}, r_{2}$ are parallel to the vector $l_{1}$. Thus, in this case,

$$
r_{1}=\beta_{1} l_{1}, \quad r_{2}=\beta_{1} l_{2}, \quad r^{\prime}=\beta_{3} l_{1} .
$$

and identity (26) being valid for $r_{i}, i=1,2$ after calculating its scalar product with $l_{1}$, casts into the form:

$$
\int_{0}^{+\infty} \frac{\left(1+\beta_{i} e^{-\alpha \tau}\right) e^{-\alpha \tau}\left\|B_{2}^{*} l_{1}\right\|^{2}}{S\left(\left|1+\beta_{i} e^{-\alpha \tau}\right| \cdot\left\|B_{2}^{*} l_{1}\right\|\right)} d \tau=0, \quad i=1,2 .
$$

The above identity 35 is impossible if $1+\beta_{i} e^{-\alpha \tau}$ is sign-definite on $[0,+\infty)$. Since $e^{-\alpha \tau}$ is strictly decreasing and $e^{-\alpha \tau} \rightarrow 0$ as $\tau \rightarrow+\infty$, we obtain that $\beta_{i}<-1, i=1,2$. By the relation $r^{\prime} \in\left[r_{1}, r_{2}\right]$ this implies that $\beta_{3}<-1$. But then there exists $\tau_{0}>0$ such that $\left|1+\beta_{3} e^{-\alpha \tau_{0}}\right| \cdot\left\|B_{2}^{*} l_{1}\right\|=0$ and this contradicts inequality 30 .

In what follows we suppose that

$$
r=m, \quad A_{22}=-I, \quad B_{2}=I .
$$

Here $I$ stands for the identity mapping of $\mathbb{R}^{m}$ onto $\mathbb{R}^{m}$.

Lemma 2. Let conditions (36) and the assumptions of Theorem 1 are satisfied. Then

$$
r_{\varepsilon} \rightarrow r_{0}=A_{12}^{*} l_{0}-2 B_{0}^{*} l_{0} \quad \text { as } \varepsilon \rightarrow 0 .
$$

Proof. Under (36), equation 20 becomes

$$
\int_{0}^{+\infty} e^{-\tau} \frac{l+e^{-\tau} r}{S\left(\left\|l+e^{-\tau} r\right\|\right)} d \tau=0
$$

where $l:=B_{0}^{*} l_{0}, r:=r_{0}+\left(A_{22}^{*}\right)^{-1} A_{12}^{*} l_{0}$. Thanks to Theorem 3 , it is sufficient to confirm that the vector $(-2 l)$ is its solution. We substitute $r=-2 l$ into the left hand side of equation (37), we obtain:

$$
\begin{aligned}
\int_{0}^{+\infty} e^{-\tau} \frac{\left(1-2 e^{-\tau}\right) l}{S\left(\mid 1-2 e^{-\tau \mid \cdot\|l\|)} d \tau\right.} & =\left[\xi=e^{-\tau}\right]=\int_{0}^{1} \frac{(1-2 \xi)}{S(|1-2 \xi| \cdot\|l\|)} d \xi l=[\eta=1-2 \xi] \\
& =\frac{1}{2} \int_{-1}^{1} \frac{\eta}{S(|\eta| \cdot\|l\|)} d \eta l=0
\end{aligned}
$$

since the integrand is odd. 
5. Asymptotic expansion of Vector $\lambda_{\varepsilon}$ Under CONDitions $(36)$

We observe that by conditions $(36)$ we have:

$$
\begin{aligned}
& B_{0}=B_{1}+A_{12}, \quad r_{0}=\left(A_{12}^{*}-2 B_{0}^{*}\right) l_{0}, \\
& C_{1, \varepsilon}^{*}(t)=B_{1}^{*} e^{A_{11}^{*} t}+A_{12}^{*}\left(e^{A_{11}^{*} t}-e^{-\frac{t}{\varepsilon}} I\right) \sum_{k=0}^{\infty}(-1)^{k} \varepsilon^{k}\left(A_{11}^{*}\right)^{k} .
\end{aligned}
$$

It follows from identities $(38)$ and $(39)$ that

$$
\begin{aligned}
C_{\varepsilon}^{*}(t) \lambda_{\varepsilon}= & C_{1,0}^{*}(t) l_{0}+C_{1,0}^{*} \Delta l-\varepsilon A_{12}^{*} e^{A_{11}^{*} t} A_{11}^{*} l_{0}- \\
& -2 e^{-\frac{t}{\varepsilon}} B_{0}^{*} l_{0}-A_{12}^{*} e^{-\frac{t}{\varepsilon}} \Delta l+\varepsilon A_{12}^{*} e^{-\frac{t}{\varepsilon}} A_{11}^{*} l_{0}+e^{-\frac{t}{\varepsilon}} \Delta r+\mathcal{F}_{2}(\varepsilon, \Delta l, \Delta r) .
\end{aligned}
$$

Here $\Delta l:=l_{\varepsilon}-l_{0}, \Delta r:=r_{\varepsilon}-r_{0}$, and $\mathcal{F}_{2}(\varepsilon, \Delta l, \Delta r)$ is a function of a second order of smallness in $\{\varepsilon, \Delta l, \Delta r\}$.

We begin with the case, when the limiting problem has a single point of the change of the type of optimal control. Suppose that for the limiting problem and the initial state of the system $x^{0}$ there exists the only moment of time $t=t_{0} \in(0, T)$ such that

$$
\begin{aligned}
& \left\|C_{1,0}^{*}(t) l_{0}\right\|<2, \quad\left\|C_{1,0}^{*}\left(t_{0}\right) l_{0}\right\|=2 \text { for all } t<t_{0}, \\
& \left\|C_{1,0}^{*}(t) l_{0}\right\|>2 \text { for all } t>t_{0}, \\
& \left.\frac{d}{d t}\left\|C_{1,0}^{*}(t) l_{0}\right\|^{2}\right|_{t=t_{0}} \neq 0 .
\end{aligned}
$$

Lemma 3. If the condition

$$
\left\|B_{0}^{*} l_{0}\right\|<2
$$

holds, then

$$
\forall l_{\varepsilon} \rightarrow l_{0} \forall r_{\varepsilon} \rightarrow\left(A_{12}^{*}-2 B_{0}^{*}\right) l_{0} \exists \varepsilon_{0}>0 \forall \varepsilon \in\left(0, \varepsilon_{0}\right) \forall t \in[0, \sqrt{\varepsilon}]\left\|C_{\varepsilon}^{*}(t) \lambda_{\varepsilon}\right\|<2 .
$$

Proof. We assume the opposite; then there exits sequences $\left\{t_{k}\right\} \subset[0, \sqrt{\varepsilon}]$ and $\left\{\varepsilon_{k}\right\}$ such that $\varepsilon_{k} \rightarrow+0$ and

$$
\left\|C_{\varepsilon_{k}}^{*}\left(t_{k}\right) \lambda_{\varepsilon_{k}}\right\| \geqslant 2 .
$$

We let $\tau_{k}:=t_{k} / \varepsilon_{k}, l_{k}:=l_{\varepsilon_{k}}, r_{k}:=r_{\varepsilon_{k}}$ and $\lambda_{k}:=\lambda_{\varepsilon_{k}}$. Then by identity (40) we get:

$$
\begin{aligned}
& C_{\varepsilon_{k}}^{*}\left(t_{k}\right) \lambda_{\varepsilon_{k}}=C_{1,0}^{*}\left(\varepsilon_{k} \tau_{k}\right) l_{0}-2 e^{-\tau_{k}} B_{0}^{*} l_{0}+\mathcal{F}_{1}\left(\varepsilon_{k}, \Delta l_{k}, \Delta r_{k}\right), \\
& \Delta l_{k}:=l_{k}-l_{0}, \quad \Delta r_{k}:=r_{k}-r_{0}, \quad \mathcal{F}_{1}\left(\varepsilon_{k}, \Delta l_{k}, \Delta r_{k}\right) \rightarrow 0 .
\end{aligned}
$$

Let $\tau_{0}$ be a limiting point of the sequence $\left\{\tau_{k}\right\}$; to shorten the notation, we suppose that $\tau_{k} \rightarrow \tau_{0}$. If $\tau_{0}=+\infty$, we pass to the limit as $k \rightarrow \infty$ in identity (45) and taking into consideration that $l_{k} \rightarrow l_{0}, r_{k} \rightarrow\left(A_{12}^{*}-2 B_{0}^{*}\right) l_{0}$, we obtain: $C_{\varepsilon_{k}}^{*}\left(\varepsilon_{k} \tau_{k}\right) \lambda_{k} \rightarrow B_{0}^{*} l_{0}$. But $\left\|B_{0}^{*} l_{0}\right\|<2$ by assumption (41) and this contradicts condition (44).

Thus, all limiting points $\tau_{0}$ are finite. Then $\varepsilon_{k} \tau_{n} \rightarrow 0$ and this is why $C_{\varepsilon_{k}}^{*}\left(\varepsilon_{k} \tau_{k}\right) \lambda_{k} \rightarrow$ $\left(1-2 e^{-\tau_{0}}\right) B_{0}^{*} l_{0}$. But

$$
\left\|\left(1-2 e^{-\tau_{0}}\right) B_{0}^{*} l_{0}\right\|=\left|1-2 e^{-\tau_{0}}\right| \cdot\left\|B_{0}^{*} l_{0}\right\| \leqslant\left\|B_{0}^{*} l_{0}\right\|<2,
$$

and this contradicts condition (44).

Theorem 4. Under condition (42), there exists $\varepsilon_{0}>0$ such that for each $\varepsilon \in\left(0, \varepsilon_{0}\right)$ there exists a single point $t_{\varepsilon}$ of the change of the type of optimal control in problem (1), that is,

$$
\left\|C_{\varepsilon}^{*}(t) \lambda_{\varepsilon}\right\|<2, \quad\left\|C_{\varepsilon}^{*}\left(t_{\varepsilon}\right) \lambda_{\varepsilon}\right\|=2 \text { for all } t<t_{\varepsilon}, \quad\left\|C_{\varepsilon}^{*}(t) \lambda_{\varepsilon}\right\|>2 \text { for all } t>t_{\varepsilon} .
$$

At that, $t_{\varepsilon} \rightarrow t_{0}$ as $\varepsilon \rightarrow 0$. 
Proof. We note that by assumption (41) there exists $\delta_{0}>0$ such that

$$
\left.\frac{d}{d t}\left\|C_{1,0}^{*}(t) l_{0}\right\|^{2}\right|_{t=t_{0}}>0 \text { for all } t \in\left[t_{0}-\delta_{0}, t_{0}+\delta_{0}\right]
$$

By (17) and (15) and since $\left\|C_{1,0}^{*}\left(t_{0}-\delta_{0}\right) l_{0}\right\|<2$ and $\left\|C_{1,0}^{*}\left(t_{0}+\delta_{0}\right) l_{0}\right\|>2$, there exists $\varepsilon_{1}>0$ such that for all $\varepsilon \in\left(0, \varepsilon_{1}\right)$ and $t \in\left[t_{0}-\delta_{0}, t_{0}+\delta_{0}\right]$ the inequalities hold:

$$
\left\|C_{\varepsilon}^{*}\left(t_{0}-\delta_{0}\right) \lambda_{\varepsilon}\right\|<2, \quad\left\|C_{\varepsilon}^{*}\left(t_{0}+\delta_{0}\right) \lambda_{\varepsilon}\right\|>2, \quad \frac{\partial}{\partial t}\left(\left\|C_{\varepsilon}^{*}(t) \lambda_{\varepsilon}\right\|^{2}\right)>0 .
$$

This implies the existence of a single point $t_{\varepsilon} \in\left[t_{0}-\delta_{0}, t_{0}+\delta_{0}\right]$ such that $\left\|C_{\varepsilon}^{*}\left(t_{\varepsilon}\right) \lambda_{\varepsilon}\right\|=2$.

Let us show that for all sufficiently small $\varepsilon>0\left(0<\varepsilon<\varepsilon_{0} \leqslant \varepsilon_{1}\right)$ there are no other points $t$ obeying identity $\left\|C_{\varepsilon}^{*}(t) \lambda_{\varepsilon}\right\|=2$.

By condition (41) there exists $\gamma>0$ such that as $\left|t-t_{0}\right| \geqslant \delta_{0}$, the estimate holds:

$$
||\left|C_{1,0}^{*}(t) l_{0} \|-2\right| \geqslant \gamma>0 .
$$

Then it follows from estimate (11) and condition (17) that for all sufficiently small $\varepsilon>0$, $t \in[\sqrt{\varepsilon}, T]$ and $\left\|t-t_{0}\right\| \geqslant \delta_{0}$ the inequality holds:

$$
||\left|C_{\varepsilon}^{*}(t) \lambda_{\varepsilon} \|-2\right| \geqslant \frac{\gamma}{2}>0 .
$$

Hence, $\left\|C_{\varepsilon}^{*}(t) \lambda_{\varepsilon}\right\| \neq 2$ for such $\varepsilon$ and $t$. On the remaining segment $[0, \sqrt{\varepsilon}]$, the relation $\left\|C_{\varepsilon}^{*}(t) \lambda_{\varepsilon}\right\| \neq 2$ holds thanks to condition (43).

Thus, in the considered case, the integral in (3) is also split into the sum of two integrals:

$$
\int_{0}^{T} \frac{C_{\varepsilon}(t) C_{\varepsilon}^{*}(t) \lambda}{S\left(\left\|C_{\varepsilon}^{*}(t) \lambda\right\|\right)} d t=\frac{1}{2} \int_{0}^{t_{\varepsilon}} C_{\varepsilon}(t) C_{\varepsilon}^{*}(t) \lambda d t+\int_{t_{\varepsilon}}^{T} C_{\varepsilon}(t) \frac{C_{\varepsilon}^{*}(t) \lambda}{\left\|C_{\varepsilon}^{*}(t) \lambda\right\|} d t .
$$

Let $\Delta l_{\varepsilon}:=l_{\varepsilon}-l_{0}, \Delta r_{\varepsilon}:=r_{\varepsilon}-r_{0}, \Delta t_{\varepsilon}:=t_{\varepsilon}-t_{0}$. Then

$$
\lambda_{\varepsilon}=\left(\begin{array}{c}
l_{0}+\Delta l_{\varepsilon} \\
\varepsilon\left(r_{0}+\Delta r_{\varepsilon}\right)
\end{array}\right), \quad \Delta l_{\varepsilon}=o(1), \quad \Delta r_{\varepsilon}=o(1), \quad \Delta t_{\varepsilon}=o(1)
$$

as $\varepsilon \rightarrow 0$, and by identities (2), (3), (46) and Theorem 4, the triple $\left\{\Delta l_{\varepsilon}, \Delta r_{\varepsilon} \Delta t_{\varepsilon}\right\}$ solves the following system of equations depending on the parameter $\varepsilon$ :

$$
\left\{\begin{aligned}
0= & F_{1}(\varepsilon, \Delta l, \Delta r, \Delta t):=-\nabla \varphi_{1}^{*}\left(-l_{\varepsilon}\right)+\nabla \varphi_{1}^{*}\left(-l_{0}\right) \\
& +\mathcal{W}_{\varepsilon}(T) y_{0}+\frac{1}{2} \int_{0}^{t_{\varepsilon}} C_{1, \varepsilon}(t) C_{\varepsilon}^{*}(t) \lambda_{\varepsilon} d t+\int_{t_{\varepsilon}}^{T} C_{1, \varepsilon}(t) \frac{C_{\varepsilon}^{*}(t) \lambda_{\varepsilon}}{\left\|C_{\varepsilon}^{*}(t) \lambda_{\varepsilon}\right\|} d t \\
0= & F_{2}(\varepsilon, \Delta l, \Delta r, \Delta t):=-\nabla \varphi_{2}^{*}\left(-\varepsilon r_{\varepsilon}\right)+\nabla \varphi_{2}^{*}(0) \\
& +\frac{1}{2} \int_{0}^{t_{\varepsilon}} \varepsilon^{-1} C_{2, \varepsilon}(t) C_{\varepsilon}^{*}(t) \lambda_{\varepsilon} d t+\int_{t_{\varepsilon}}^{T} \varepsilon^{-1} C_{2, \varepsilon}(t) \frac{C_{\varepsilon}^{*}(t) \lambda_{\varepsilon}}{\left\|C_{\varepsilon}^{*}(t) \lambda_{\varepsilon}\right\|} d t \\
0= & G(\varepsilon, \Delta l, \Delta r, \Delta t):=\left\|C_{\varepsilon}^{*}(t+\Delta t) \lambda_{\varepsilon}\right\|^{2}-\left\|C_{1,0}^{*}\left(t_{0}\right) l_{0}\right\|^{2} .
\end{aligned}\right.
$$

We note that the functions $F_{1}, F_{2}$ and $G$ are continuous, and $G$ is infinitely differentiable. Let us study their asymptotic expansions with respect to infinitesimals $\Delta l, \Delta r$ and $\Delta t$. 
By the infinite differentiability of the functions $\varphi_{1}^{*}$ and $\varphi_{2}^{*}$ and in view of identity $\varphi_{2}^{*}(0)=0$ we obtain:

$$
\begin{aligned}
& -\nabla \varphi_{1}^{*}\left(-l_{0}-\Delta l\right)+\nabla \varphi_{1}^{*}\left(-l_{0}\right) \sim D^{2} \varphi_{1}^{*}\left(-l_{0}\right) \Delta l+\sum_{k=2}^{\infty} \Phi_{1, k}(\Delta l), \\
& -\nabla \varphi_{2}^{*}\left(-\varepsilon r_{\varepsilon}\right)+\nabla \varphi_{2}^{*}(0) \sim D^{2} \varphi_{2}^{*}(0) r_{0} \varepsilon+\sum_{k=2}^{\infty} \Phi_{2, k}(\varepsilon, \Delta r),
\end{aligned}
$$

where $D^{2} \varphi_{1}^{*}\left(-l_{0}\right)$ and $D^{2} \varphi_{2}^{*}(0)$ are second order differentials of $\varphi_{1}^{*}$ and $\varphi_{2}^{*}$ at the points $\left(-l_{0}\right)$ and 0 , respectively, and $\Phi_{1, k}(\Delta l)$ and $\Phi_{2, k}(\varepsilon, \Delta l)$ are homogeneous functions of order $k$, namely, polynomials of the components of the vector $\Delta l$ and $\varepsilon$.

By identity (7),

$$
\mathcal{W}_{\varepsilon}(T) y_{0} \sim \varepsilon e^{A_{11} T} A_{12} y_{0}+\sum_{k=2}^{\infty} \varepsilon^{k} y_{k}
$$

where $y_{k}$ are known vectors.

We split each integral in the first and second identity in system of equations (47) into two parts

$$
\int_{0}^{t_{0}+\Delta t}=\int_{0}^{t_{0}}+\int_{t_{0}}^{t_{0}+\Delta t}, \quad \int_{t_{0}+\Delta t}^{T}=\int_{t_{0}+\Delta t}^{t_{0}}+\int_{t_{0}}^{T}
$$

and we denote the integrals by $I_{1}(\varepsilon, \Delta \lambda), I_{2}(\varepsilon, \Delta \lambda), I_{3}(\varepsilon, \Delta \lambda)$ and $I_{4}(\varepsilon, \Delta \lambda)$, respectively.

We note that by identity (7), the asymptotics of integrands in $I_{2}-I_{4}$ is power in $\varepsilon$ and the components of the vector $\Delta \lambda$ with coefficients smoothly depending on $t$.

To expand the integrals $I_{2}$ and $I_{3}$ in $\Delta t$, we should additionally expand the coefficients depending on $t$ into the Taylor series at the point $t_{0}$ and to integrate the obtained expansions over the mentioned segments.

We observe that in $I_{2}$ and $I_{3}$, the terms of the first order of smallness in $\Delta t$ are of the form:

$$
\frac{C_{1,0}\left(t_{0}\right) C_{1,0}^{*}\left(t_{0}\right) l_{0}}{2} \Delta t, \quad-\frac{C_{1,0}\left(t_{0}\right) C_{1,0}^{*}\left(t_{0}\right) l_{0}}{\left\|C_{1,0}^{*}\left(t_{0}\right) l_{0}\right\|} \Delta t
$$

respectively. Since

$$
\left\|C_{1,0}^{*}\left(t_{0}\right) l_{0}\right\|=2, \quad I_{2}(\varepsilon, \Delta \lambda)=O(\Delta t), \quad I_{2}(\varepsilon, \Delta \lambda)=O(\Delta t),
$$

the expansions of the $I_{2}+I_{3}$ contains no terms of the first order of smallness in $\Delta l, \Delta r, \Delta t$ and $\varepsilon$.

By estimate (14) and identity (39), on $\left[t_{0}, T\right]$ we have asymptotic identities:

$$
C_{1, \varepsilon}^{*}(t)=B_{1}^{*}(t) e^{A_{11}^{*} t}+A_{12}^{*} e^{A_{11}^{*} t} \sum_{k=0}^{\infty}(-1)^{k} \varepsilon^{k}\left(A_{11}^{*}\right)^{k}, \quad C_{2, \varepsilon}^{*}(t)=\mathbb{O} \quad \text { as } \quad \varepsilon \rightarrow 0 .
$$

Hence,

$$
\begin{aligned}
\frac{1}{2} \int_{0}^{t_{\varepsilon}} \varepsilon^{-1} C_{2, \varepsilon}(t) C_{\varepsilon}^{*}(t) \lambda_{\varepsilon} d t+\int_{t_{\varepsilon}}^{T} \varepsilon^{-1} C_{2, \varepsilon}(t) \frac{C_{\varepsilon}^{*}(t) \lambda_{\varepsilon}}{\left\|C_{\varepsilon}^{*}(t) \lambda_{\varepsilon}\right\|} d t & =\frac{1}{2} \int_{0}^{t_{0}} \varepsilon^{-1} C_{2, \varepsilon}(t) C_{\varepsilon}^{*}(t) \lambda_{\varepsilon} d t+\mathbb{O} \\
& =: I_{5}(\varepsilon, \Delta \lambda)+\mathbb{O},
\end{aligned}
$$

while the power asymptotics of the integrals $I_{i}, i=2,3,4$ contains no $\Delta r$.

We introduce the notation: $\left(I_{i}(\varepsilon, \Delta \lambda)\right)_{1}$ is a linear in $\Delta l, \Delta r, \Delta t$ and $\varepsilon$ part of the integral $I_{i}(\varepsilon, \Delta \lambda)$. 
By Theorem 4, identities (50) and

$$
\int_{0}^{t_{0}} e^{-\frac{t}{\varepsilon}} f\left(t, l_{\varepsilon}, r_{\varepsilon}\right) d t=O(\varepsilon)
$$

if $f\left(t, l_{\varepsilon}, r_{\varepsilon}\right)$ is uniformly bounded on $\left[0, t_{0}\right]$, by simple calculations we get:

$$
\begin{aligned}
\left(I_{1}(\varepsilon, \Delta \lambda)\right)_{1}= & \frac{1}{2} \int_{0}^{t_{0}} C_{1,0}(t) C_{1,0}^{*}(t) d t \Delta l+\varepsilon f_{1}=: D_{11} \Delta l+\varepsilon f_{1}, \\
\left(I_{3}(\varepsilon, \Delta \lambda)\right)_{1}= & \int_{t_{0}}^{T} C_{1,0}(t) \frac{C_{1,0}^{*}(t) \Delta l\left\|C_{1,0}^{*}(t) l_{0}\right\|^{2}-\left\langle C_{1,0}^{*}(t) \Delta l, C_{1,0}^{*}(t) l_{0}\right\rangle C_{1,0}^{*}(t) l_{0}}{\left\|C_{1,0}^{*}(t) l_{0}\right\|^{3}} d t \\
& +\varepsilon f_{3}=: D_{12} \Delta l+\varepsilon f_{3}, \\
\left(I_{5}(\varepsilon, \Delta \lambda)\right)_{1}= & \frac{1}{4} \Delta r+\frac{1}{4}\left(2 B_{0}^{*}-A_{12}^{*}\right) \Delta l+\varepsilon f_{5},
\end{aligned}
$$

where $f_{1}, f_{3}$ and $f_{5}$ are uniquely calculated by $l_{0}$. At that, by assumption (36) and CauchySchwarz inequality we have:

$$
D_{11}>0, \quad D_{12} \geqslant 0 .
$$

By identity (50) we can find the asymptotics for the function $G(\varepsilon, \Delta l, \Delta t)$ as $\Delta l, \Delta t$ and $\varepsilon$ tend to zero:

$$
\begin{aligned}
G(\varepsilon, \Delta l, \Delta t) \sim & 2\left\langle C_{1,0}^{*}\left(t_{0}\right) l_{0}, C_{1,0}^{*}\left(t_{0}\right) \Delta l+\left(C_{1,0}^{*}\right)^{\prime}\left(t_{0}\right) l_{0} \Delta t+\varepsilon A_{11}^{*} e^{A_{11}^{*} t_{0}} l_{0}\right\rangle \\
& +\sum_{k=2}^{\infty} G_{k}(\varepsilon, \Delta l, \Delta t), \quad\left(C_{1,0}^{*}\right)^{\prime}\left(t_{0}\right):=\left.\frac{d}{d t} C_{1,0}^{*}(t)\right|_{t=t_{0}},
\end{aligned}
$$

where $G_{k}(\varepsilon, \Delta l, \Delta t)$ are some homogeneous functions of order $k$ in $\varepsilon$ and the components of the vectors $\Delta l$ and $\Delta r$.

Thus, by identities (48), (49), (51)-(53) and (55), the system for the first corrector of (47) reads as

$$
\left\{\begin{array}{l}
\varepsilon g_{1}=D^{2} \varphi_{1}^{*}\left(-l_{0}\right) \Delta l_{1}+D_{11} \Delta l_{1}+D_{12} \Delta l_{1} \\
\varepsilon g_{2}=\frac{1}{4} \Delta r_{1}+\frac{1}{4}\left(2 B_{0}^{*}-A_{12}^{*}\right) \Delta l_{1} \\
\varepsilon g_{3}=2\left\langle C_{1,0}^{*}\left(t_{0}\right) l_{0}, C_{1,0}^{*}\left(t_{0}\right) \Delta l_{1}\right\rangle+\left\langle C_{1,0}^{*}\left(t_{0}\right) l_{0},\left(C_{1,0}^{*}\right)^{\prime}\left(t_{0}\right) l_{0}\right\rangle \Delta t_{1} .
\end{array}\right.
$$

By the convexity of $\varphi_{1}$ and inequalities (54), we have

$$
D^{2} \varphi_{1}^{*}\left(-l_{0}\right)+D_{11}+D_{12}>0,
$$

and this is why the first equation in system (56) determines uniquely $\Delta l_{1}=\varepsilon l_{1}$. After that by the second equation in system (56) we uniquely find $\Delta r_{1}=\varepsilon r_{1}$. Finally, by conditions (41), the coefficient at $\Delta t_{1}$ is non-zero and hence, by the third equation in system (56) we uniquely determine $\Delta t_{1}=\varepsilon t_{1}$. Thus, the linear operator of the first corrector for system (56), that is, the operator

$$
\mathcal{D}\left(\begin{array}{c}
\Delta l_{1} \\
\Delta r_{1} \\
\Delta t_{1}
\end{array}\right)=\left(\begin{array}{c}
D^{2} \varphi_{1}^{*}\left(-l_{0}\right) \Delta l_{1}+D_{11} \Delta l_{1}+D_{12} \Delta l_{1} \\
\frac{1}{4} \Delta r_{1}+\frac{1}{4}\left(2 B_{0}^{*}-A_{12}^{*}\right) \Delta l_{1} \\
2\left\langle C_{1,0}^{*}\left(t_{0}\right) l_{0}, C_{1,0}^{*}\left(t_{0}\right) \Delta l_{1}\right\rangle+\left\langle C_{1,0}^{*}\left(t_{0}\right) l_{0},\left(C_{1,0}^{*}\right)^{\prime}\left(t_{0}\right) l_{0}\right\rangle \Delta t_{1}
\end{array}\right)
$$

is continuously invertible. 
The process of determining next terms in the expansions of $\Delta l, \Delta r$ and $\Delta t$ is continued in a standard way. Assume that we have approximations of $\Delta l, \Delta r$ and $\Delta t$ up to $N$ th order. Then the quantities

$$
\Delta l_{N+1}:=\Delta l-\sum_{k=1}^{N} \varepsilon^{k} l_{k}, \quad \Delta r_{N+1}:=\Delta r-\sum_{k=1}^{N} \varepsilon^{k} r_{k}, \quad \Delta t_{N+1}:=\Delta t-\sum_{k=1}^{N} \varepsilon^{k} t_{k}
$$

satisfy the relations

$$
\mathcal{D}\left(\begin{array}{c}
\Delta l_{N+1} \\
\Delta r_{N+1} \\
\Delta t_{N+1}
\end{array}\right)=O\left(\varepsilon^{N+1}\right)+O\left(\varepsilon\left\|z_{N+1}\right\|\right)+O\left(\left\|z_{N+1}\right\|^{2}\right), \quad z_{N+1}:=\left(\begin{array}{c}
\Delta l_{N+1} \\
\Delta r_{N+1} \\
\Delta t_{N+1}
\end{array}\right) .
$$

By the continuous invertibility of the operator $\mathcal{D}$, by relations (57) we obtain:

$$
z_{N+1}=O\left(\varepsilon^{N+1}\right)+O\left(\varepsilon\left\|z_{N+1}\right\|\right)+O\left(\left\|z_{N+1}\right\|^{2}\right) .
$$

As it was shown in [10, Stat. 2], it follows from (58) that $z_{N+1}=O\left(\varepsilon^{N+1}\right)$. Thus, we have proved the following theorem.

Theorem 5. Let Assumptions 2 and 3 be satisfied as well as conditions (41) and (42). Then the vectors $l_{\varepsilon}, r_{\varepsilon}$ and the moment of time $t_{\varepsilon}$ are expanded into power asymptotic series

$$
l_{\varepsilon} \stackrel{a s}{=} l_{0}+\sum_{k=1}^{\infty} \varepsilon^{k} l_{k}, \quad r_{\varepsilon} \stackrel{a s}{=}\left(A_{12}^{*}-2 B_{0}^{*}\right) l_{0}+\sum_{k=1}^{\infty} \varepsilon^{k} r_{k}, \quad t_{\varepsilon} \stackrel{a s}{=} t_{0}+\sum_{k=1}^{\infty} \varepsilon^{k} t_{k}, \quad \varepsilon \rightarrow 0,
$$

whose coefficients can be found in a recurrent way.

Similar results are true in a more general case, when there exist finitely many points $\left\{t_{1}, t_{2}, \ldots, t_{p}\right\} \subset(0, T)$ such that

$$
\left\|C_{0}^{*}(t) l_{0}\right\| \neq 2, \quad\left\|C_{0}^{*}\left(t_{i}\right) l_{0}\right\|^{2}=4,\left.\quad \frac{d}{d t}\left\|C_{0}^{*}\left(t_{i}\right) l_{0}\right\|^{2}\right|_{t=t_{i}} \neq 0
$$

for all $t \in[0, T] \backslash\left\{t_{i}\right\}_{i=1}^{p}$ and condition $(42)$ holds true.

In this case an analogue of Theorem 4 reads as follows.

Theorem 6. Let Assumptions (36), (42) and (59) hold true. Then there exists $\varepsilon_{0}>0$ such that for each $\varepsilon \in\left(0, \varepsilon_{0}\right)$ there exist the points $\left\{t_{1, \varepsilon}, t_{2, \varepsilon}, \ldots, t_{p, \varepsilon}\right\} \subset(0, T)$ of the change of the type of optimal control in problem (1). There are no other points of the change of the type of optimal control and $t_{i, \varepsilon} \rightarrow t_{i}$ as $\varepsilon \rightarrow 0$ for each $i=1, \ldots, p$.

The proof of this theorem is similar to that of Theorem 4.

We note that in this case the system of equations similar to system (47) contains a set of $p$ equations $0=G_{p}$ instead of one scalar equation $0=G$; these equations correspond to the points $t_{i, \varepsilon}$ and the unknowns are $\Delta l, \Delta r$ and $\Delta t_{i}, i=1, \ldots, p$.

Similar to Theorem 5, we can prove the following final theorem.

Theorem 7. Let Assumptions 2 and 3 are satisfied as well as conditions (36), (42) and (59). Then the vectors $l_{\varepsilon}, r_{\varepsilon}$ and the moments of time $\left\{t_{1, \varepsilon}, t_{2, \varepsilon}, \ldots, t_{p, \varepsilon}\right\}$ are expanded into power asymptotic series

$$
\begin{aligned}
& l_{\varepsilon} \stackrel{a s}{=} l_{0}+\sum_{k=1}^{\infty} \varepsilon^{k} l_{k}, \quad r_{\varepsilon} \stackrel{a s}{=}\left(A_{12}^{*}-2 B_{0}^{*}\right) l_{0}+\sum_{k=1}^{\infty} \varepsilon^{k} r_{k}, \\
& t_{i, \varepsilon} \stackrel{a s}{=} t_{i}+\sum_{k=1}^{\infty} \varepsilon^{k} t_{i, k}, \quad i=1, \ldots, p, \quad \varepsilon \rightarrow 0,
\end{aligned}
$$

whose coefficients can be found in a recurrent way. 


\section{BIBLIOGRAPHY}

1. L.S. Pontryagin, V.G. Boltyanskii, R.V. Gamkrelidze, E.F. Mishchenko. The mathematical theory of optimal processes. Fizmatgiz, Moscow (1961). [Interscience Publ. John Wiley and Sons. Inc., New York (1962).]

2. N.N. Krasovskii. Theory of motion control. Linear systems. Nauka, Moscow (1968). (in Russian).

3. E.B. Lee, L. Markus. Foundations of optimal control theory. John Wiley and Sons. Inc., New York (1967).

4. A.B. Vasil'eva, M.G. Dmitriev. Singular perturbations in problems of optimal control // Itogi Nauki Tekhn. Ser. Mat. Anal. 20, 3-77 (1982). [J. Soviet Math. 34:3, 1579-1629 (1986).]

5. P.V. Kokotovic, A.H. Haddad. Controllability and time-optimal control of systems with slow and fast modes // IEEE Trans. Automat. Control. 20:1, 111-113 (1975).

6. A.L. Dontchev. Perturbations, approximations and sensitivity analisis of optimal control systems. Mir, Moscow (1987). [Springer-Verlag, Berlin (1983).]

7. A.I. Kalinin, K.V. Semenov. Asymptotic optimization method for linear singularly perturbed systems with multidimensional control // Zhurn. Vychisl. Matem. Matem. Fiz. 44:3, 432-443 (2004). [Comp. Math. Math. Phys. 44:3, 407-417 (2004).]

8. A.R. Danilin, Yu.V. Parysheva. Asymptotics of the optimal cost functional in a linear optimal control problem // Dokl. AN. 427:2, 151-154 (2009). [Dokl. Math. 80:1, 478-481 (2009).]

9. A.R. Danilin, O.O. Kovrizhnykh. Time-optimal control of a small mass point without environmental resistance // Dokl. RAN. 451:6, 612-614 (2013). [Dokl. Math. 88:1, 465-467 (2013).]

10. A.A. Shaburov. Asymptotic expansion of a solution to a singularly perturbed optimal control problem with a convex integral performance index whose terminal part depends on slow variables only // Trudy IMM UrO RAN. 24:2, 280-289 (2018). (in Russian).

11. R. Rockafellar. Convex analysis. Princeton University Press, Princeton (1970).

Aleksei Rufimovich Danilin,

Institute of Mathematics and Mechanics,

Ural Branch of Russian Academy of Sciences,

Sofia Kovalevskaya str. 16,

620990, Ekaterinburg, Russia

E-mail: dar@imm.uran.ru

Alexandr Alexandrovich Shaburov,

Ural Federal University,

Mir str. 19,

620002, Ekaterinburg, Russia

E-mail: alexandershaburov@mail.ru 